\title{
Luminosity Increase at the Incoherent Beam-Beam Limit with Six Superbunches in RHIC ${ }^{1}$
}

\author{
W. Fischer and M. Blaskiewicz
}

Brookhaven National Laboratory, Upton, New York 11973

\begin{abstract}
By colliding bunches of greater length under a larger angle, the tune spread caused by the beam-beam interaction can be reduced. Assuming a constant limit for the beam-beam tune shift, the bunch intensity can then be raised. In this way, a luminosity increase is possible. We review this strategy for proton beams in RHIC, with two collisions and consider six long bunches. Barrier cavities are used to fill every accelerating bucket of the machine, except for an abort gap, and to create the superbunches bunches at store. Resonances driven by the beam-beam interaction and coherent effects are neglected in this article.
\end{abstract}

\section{INTRODUCTION}

Luminosity limits set by the incoherent beam-beam tune shift were discussed for unbunched beams by Keil [1]. He showed that an increase in the crossing angle reduces the beam-beam tune shift and allows a higher line density, which in turn leads to an increased luminosity. Recently, Ruggiero and Zimmermann extended this analysis to bunched beams [2]. With one horizontal and one vertical collision under the same angle, the beam-beam tune spread in both planes is the same for round beams.

Extremely long bunches, called superbunches, are the basis of a recently proposed hadron collider concept [3]. In this proposal, beam is stacked in very long bunches using barrier cavities, and accelerated with an induction device [4].

In this article we estimate the luminosity for six very long bunches in RHIC given a certain limit for the incoherent beam-beam tune spread. With six symmetrically distributed superbunches any two of the RHIC experiments can be served with luminosity. For the scheme under investigation here, barrier cavities are needed for injection and for the gap maintenance at store. Acceleration is done with the existing $28 \mathrm{MHz}$ system with harmonic number $h=360$ [5]. In an earlier article [6] we considered bunches in the RHIC accelerating and storage buckets, as well a superbunches that fill the whole circumference except for an abort gap.

Basic parameters are summarized in Tab. 1. We assume that a total tune spread of $\Delta Q_{\max }=-0.03$ can be accomodated, caused by one horizontal and one vertical crossing. This is consistent with the maximum values

\footnotetext{
1 Work supported by US DOE, contract No DE-AC02-98CH10886.
}

achieved in the SPS and Tevatron, but challenging for routine operation.

The crossing angle $\theta$ is measured as the full angle from one beam to the other. With the current vertical corrector strength, a crossing angle of $0.84 \mathrm{mrad}$ can be implemented at store [7]. However, some of this strength may be needed to correct for unwanted orbit effects. We therefore assume that vertical crossing angles of $0.5 \mathrm{mrad}$ can be implemented with the existing hardware. Larger horizontal crossing angles were used in the past.

We take for the length, in which the beam-beam force is active, the distance between the DX beam splitting magnets. Once the beams reach these magnets they are quickly separated. The effective detector length, the region in which collisions are recorded, is the largest length currently used by any one of the RHIC detectors [8].

We neglect here resonances driven by the beam-beam interaction, coherent effects and end effects of the superbunches. However, we note that large crossing angles can be beneficial in damping coherent beam-beam modes [9]. Furthermore, it is assumed that the long-range beam-beam interactions during the energy ramp do not lead to significant emittance increases or beam losses.

\section{BEAM PREPARATION}

At injection a long bunch that almost fills the circumference, except for an abort gap, is maintained by a barrier cavity. New bunches are injected into buckets that are then merged with the existing single superbunch. In this way, an amount of beam can be injected much larger than currently possible.

When the injection is finished the accelerating system is turned on, and the beam is captured in all the $28 \mathrm{MHz}$ buckets, except for the abort gap. We assume that $4 \cdot 10^{11}$ 
TABLE 1. Parameters for acceleration and superbunches.

\begin{tabular}{lccc}
\hline quantity & unit & $\begin{array}{c}\text { accel. } \\
\text { bunch }\end{array}$ & $\begin{array}{c}\text { super } \\
\text { bunch }\end{array}$ \\
\hline circumference $C$ & $\mathrm{~m}$ & \multicolumn{2}{c}{3833} \\
beam-beam limit $\Delta Q_{\max }$ & $\ldots$ & \multicolumn{2}{c}{-0.03} \\
crossing angle $\theta$ & $\mathrm{mrad}$ & \multicolumn{2}{c}{0.5} \\
lattice $\beta^{*}$ at store & $\mathrm{m}$ & \multicolumn{2}{c}{1.0} \\
relativistic $\gamma$ at store & $\ldots$ & \multicolumn{2}{c}{260} \\
emittance $\varepsilon_{N}, 95 \%$ & $\mu \mathrm{m}$ & \multicolumn{2}{c}{20} \\
interaction region length $l$ & $\mathrm{~m}$ & \multicolumn{2}{c}{20} \\
eff. detector length $l_{\text {det }}$ & $\mathrm{m}$ & \multicolumn{2}{c}{0.7} \\
particles per bunch $N_{b}$ & $10^{11}$ & 4.0 & 215 \\
number of bunches $n_{b}$ & $\ldots$ & 320 & \multicolumn{2}{c}{6} \\
bunch area $S, 95 \%$ & $\mathrm{eV} \cdot \mathrm{s}$ & 1.0 & \multicolumn{2}{c}{$\ldots$} \\
rf frequency $f_{r f}$ & $\mathrm{MHz}$ & 28 & $\ldots$ \\
gap voltage $V_{g a p}$ & $\mathrm{MV}$ & 0.3 & $\ldots$ \\
rms bunch length $\sigma_{z}$ & $\mathrm{~m}$ & 0.45 & $\ldots$ \\
luminosity $L$ & $10^{33} \mathrm{~cm}^{-2} \mathrm{~s}^{-1}$ & 1.5 & 2.3 \\
\hline
\end{tabular}

protons can be accelerated in 320 of the 360 accelerating buckets. During acceleration the beams are vertically separated in the interaction regions. Bunches experience 5 parasitic collisions in every interaction region [10], a total of 30 per turn. It is possible to provide a separation of at least 7 transverse rms beam sizes. Operational experience so far has shown that the beam-beam effects cannot be completely suppressed in this way. Compared to current running conditions, the beam-beam effect may be mitigated by a larger transverse separation and better tune control along the ramp. We assume here that the beam can be accelerated without significant emittance growth or beam loss.

At store the beam is then transferred into six long bunches that are maintained by barrier cavities. The length of the superbunches is determined by the maximum line density that can be sustained at the beam-beam limit given a certain crossing angle.

\section{SUPERBUNCH GAP MAINTENANCE}

In this section the maintenance of the six superbunches with barrier cavities is discussed. Experience with and plans for barrier cavities are reported in Refs. $[4,12,13$, 14].

Let $\varepsilon=E-E_{s}$ denote the energy deviation for a given particle and let $\tau$ denote its arrival time with respect to the synchronous particle. Using turn number $n$ as the time-like variable the equations for $\tau$ and $\varepsilon$ are

$$
\begin{aligned}
& \frac{d \varepsilon}{d n}=-q V_{s}+q V_{r f}(\tau), \\
& \frac{d \tau}{d n}=T_{r e v} \eta \frac{\varepsilon}{\beta^{2} E_{s}},
\end{aligned}
$$

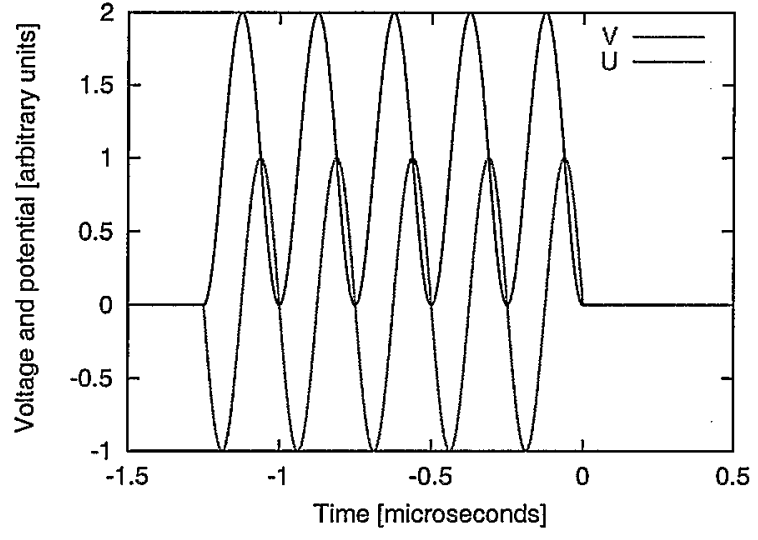

FIGURE 1. Voltage $V$, and potential $U$ waveforms of a barrier cavity with $f_{r f}=4 \mathrm{MHz}$.

where $q$ is the particle charge, $V$ the rf voltage, $T_{\text {rev }}$ the revolution time, $\eta$ the slip factor and $\beta$ the relativistic beam parameter. The subscript $s$ denotes the synchronous particle. Eqs. (1) and (2) correspond to the Hamiltonian

$$
H(\tau, \varepsilon)=\frac{T_{r e v} \eta}{2} \frac{\varepsilon^{2}}{\beta^{2} E_{s}}+q V_{s} \tau-q \int_{0}^{\tau} V_{r f}\left(\tau_{1}\right) d \tau_{1} .
$$

For adiabatic processes the phase space density is constant on curves of constant $H(\varepsilon, \tau)$. For these a dimensionless potential energy $U(\tau)$ can be defined by

$$
U(\tau)=\frac{2 \beta^{2}}{\eta T_{\text {rev }}\left(E_{s} / q\right)}\left[V_{s} \tau-\int_{0}^{\tau} V_{r f}\left(\tau_{1}\right) d \tau_{1}\right]
$$

with which the maximum energy deviation on a given contour $\hat{\varepsilon}=\left(E-E_{s}\right)_{\max }$ can be written as

$$
\frac{\hat{\varepsilon}^{2}}{E_{s}^{2}}=\frac{\varepsilon^{2}}{E_{s}^{2}}+U(\tau) \text {. }
$$

We choose $V_{r f}$ so that $U(\tau) \geq 0$. With Eqs. (4) and (5) the potential and $\mathrm{rf}$ voltage for a given energy deviation $\hat{\varepsilon}$ can be determined for a given waveform of the barrier cavity voltage.

For gap maintenance we have $V_{s}=0$. A gap between the bunches of $1 \mu \mathrm{s}$ length can be created, for example, by one waveform of a $f_{r f}=1 \mathrm{MHz}$ rf system [13]. In this way about half of the RHIC circumference can be filled with beam in six superbunches. A gap of $1 \mu$ s length would also be sufficient as an abort gap. For shorter gaps between bunches, a higher frequency is needed. The voltage and potential waveforms for such a system are illustrated in Fig. 1, where a sinusoidal waveform for the voltage is assumed, $V(t)=-\hat{V} \sin \left(2 \pi f_{r f} t\right)$. The peak voltage $\hat{V}$ as a function of the energy spread $\hat{\varepsilon}$ can be obtained from Eq. (4) as

$$
\hat{V}=\frac{\pi}{2} \frac{\eta T_{r e v} f_{r f}}{\beta^{2}} \frac{E_{s}}{q} \frac{\hat{\varepsilon}^{2}}{E_{s}^{2}}
$$


TABLE 2. Rf parameters at injection and storage.

\begin{tabular}{lccc}
\hline quantity & unit & injection & storage \\
\hline relativistic $\gamma$ & $\ldots$ & 26 & 260 \\
kinetic energy $E_{k}$ & $\mathrm{GeV}$ & 23.4 & 243.0 \\
slip factor $\eta$ & $\ldots$ & 0.00044 & 0.00191 \\
energy spread $\hat{\varepsilon}$ & $\ldots$ & $10^{-3}$ & $10^{-3}$ \\
barrier frequency $f_{r f}$ & $\mathrm{MHz}$ & 1.0 & 1.0 \\
gap voltage $\hat{V}$ & $\mathrm{kV}$ & 0.2 & 9 \\
\hline
\end{tabular}

With an energy spread of $\hat{\varepsilon} / E_{s}=10^{-3}$ and a frequency of $f_{r f}=1 \mathrm{MHz}$ the peak voltage needed at injection and storage is $0.2 \mathrm{kV}$ and $9 \mathrm{kV}$ respectively (see Tab Tab. 2). Previous barrier cavity work has created $10 \mathrm{kV}$ single period sine waves using a single cavity [13]. Thus gap maintenance appears possible.

\section{LUMINOSITY}

In Ref. [2] formulas are given for the incoherent tune shift due to the beam-beam interaction for particles in the beam center, and for the luminosity. For the conditions given in Tab. 1 the luminosity per interaction point is $L=2.3 \cdot 10^{33} \mathrm{~cm}^{-2} \mathrm{~s}^{-1}$ with six superbunches. This about two orders of magnitude larger than the luminostiy under current running conditions $L=2.7 \cdot 10^{31} \mathrm{~cm}^{-2} \mathrm{~s}^{-1}\left(N_{b}=\right.$ $10^{11}, n_{b}=55$ ). In Tab. 1 also given is the luminosity for colliding the 320 acceleration bunches, $L=1.5$. $10^{31} \mathrm{~cm}^{-2} \mathrm{~s}^{-1}$. In this case the large number of parasitic collisions needs to be analyzed. With six superbunches the luminosity is about $50 \%$ higher than with the bunched beam.

We now show the change of the superbunch length and luminosity per interaction point under variation of the crossing angle $\theta$, intensity of the acceleration buckets $N_{b}$, and the sustainable total beam-beam tune spread $\Delta Q_{m i n}$.

In Fig. 2 the variation is shown for the crossing angle $\theta$. With small crossing angles the superbunches become very long. With crossing angles below $0.2 \mathrm{mrad}$ the whole ring would be filled. With crossing angles larger than $0.5 \mathrm{mrad}$ the luminosity increase slowes down.

At the beam-beam limit the achievable luminosity is proportional to the bunch intensity and the beam-beam tune shift $\Delta Q_{\max }$. It is not dependent on the emittance since both the beam-beam tune shift and the luminosity are inversely proportional to the emittance.

For superbunches and crossing angles $\theta \ll 1$ one has $[1,2]$

$$
L=\frac{\gamma N_{b} n_{b}}{\beta^{*}}\left|\Delta Q_{\max }\right| F\left(\theta, l, l_{\text {det }}\right)
$$

where the form factor $F\left(\theta, l, l_{d e t}\right)$ is fixed for a certain configuration of $\left(\theta, l, l_{d e t}\right)$. The linear dependence of the luminosity on the bunch intensity $N_{b}$ can be seen in Fig. 3, and on the beam-beam tune shift $\Delta Q_{\max }$ in Fig. 4.

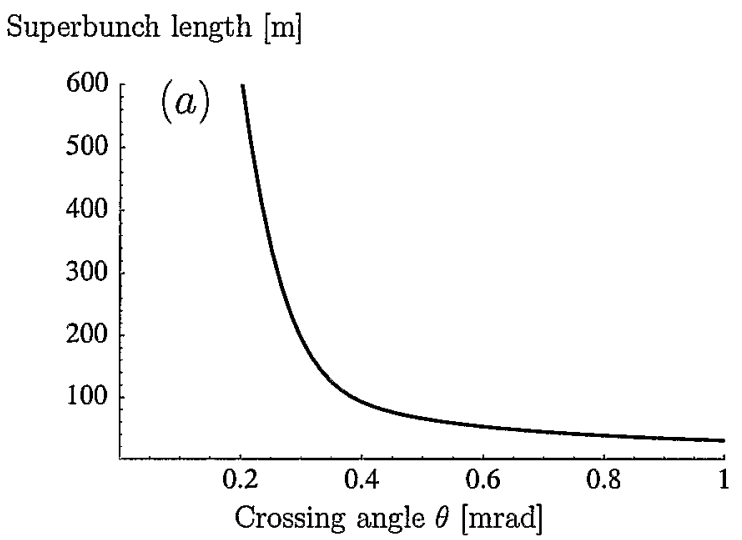

Luminosity $\left[\mathrm{cm}^{-2} \mathrm{~s}^{-1}\right]$

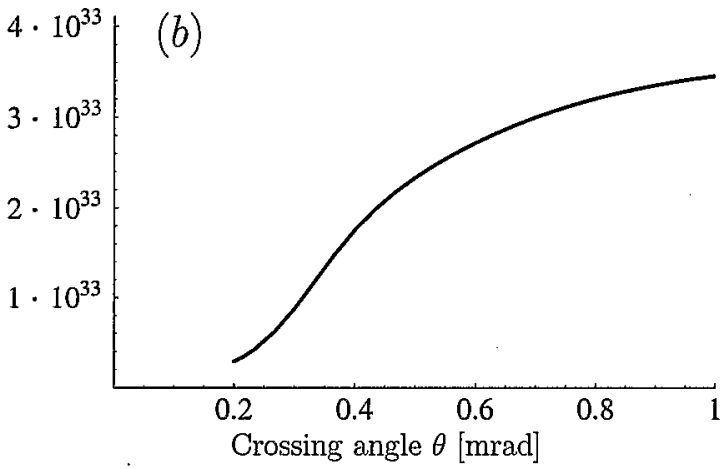

FIGURE 2. Superbunch length and luminosity per interaction point as a function of the crossing angle $\theta$ in parts (a) and (b) respectively. Other parameters are given in Tab. 1.

\section{SUMMARY}

We estimated the achievable luminosity with six superbunches in RHIC for the incoherent beam-beam spread of $\Delta Q_{\max }=-0.03$. The estimated luminosity of $L=2.3$. $10^{33} \mathrm{~cm}^{-2} \mathrm{~s}^{-1}$ is about two orders of magnitude larger than the luminosity under current running conditions, and about 50\% higher than for bunches with the same total intensity. For the preparation of six superbunches at store, barrier cavities are needed with parameters close to those that were demonstrated in the past.

A number of effects were neglected in this study. Among those are resonant effects, coherent effect, end effects of the superbunches, and long-range beam-beam interactions on the energy ramp. These effects will reduce the estimated luminosity. Furthermore, a number of system changes will be needed [15].

\section{ACKNOWLEDGMENTS}

The authors are thankful for discussions with J.M. Brennan, M. Harrison, W. MacKay, T. Roser, F. Ruggiero, S. Peggs, V. Ptitsyn, K. Takayama, and F. Zimmermann. 
Superbunch length $[\mathrm{m}]$

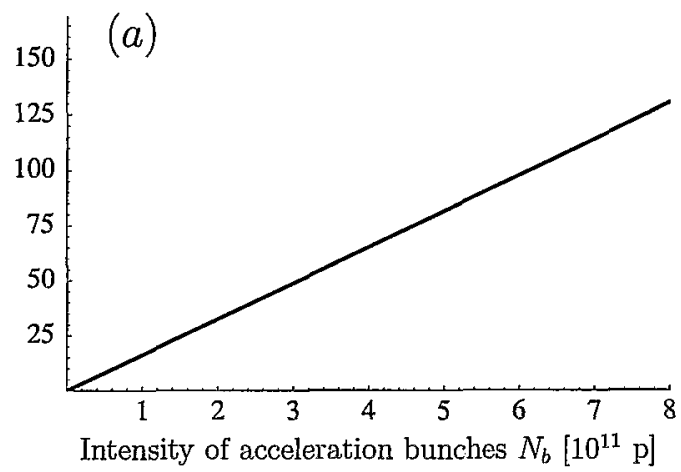

Luminosity $\left[\mathrm{cm}^{-2} \mathrm{~s}^{-1}\right]$

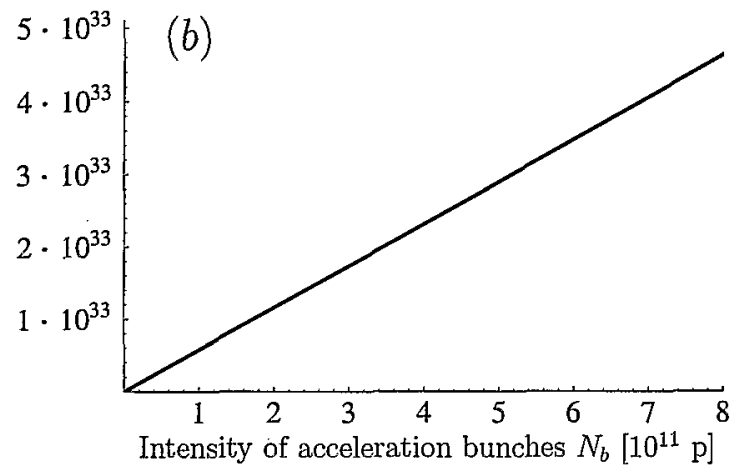

FIGURE 3. Superbunch length and luminosity per interaction point as a function of the bunch intensity $N_{b}$ of the accelerated bunches in parts (a) and (b) respectively. Other parameters are given in Tab. 1 .

\section{REFERENCES}

1. E. Keil, "Luminosity optimization for storage rings with low- $\beta$ sections and small crossing angles", Nucl. Instrum. Methods 113, 333 (1973).

2. F. Ruggiero and F. Zimmermann, "Luminosity optimization near the beam-beam limit by increasing bunch length or crossing angle", Phys. Rev. ST Accel. Beams 5061001 (2002).

3. K. Takayama, J. Kishiro, M. Sakuda, Y. Shimosaki, and M. Wake, "Superbunch hadron colliders", Phys. Rev. Lett. Vol. 88 No. 14 (2002).

4. J. Kishiro and K. Takayama, "Induction synchrotron", Nucl.Inst. Meth. A451, 304-317 (2000).

5. H. Hahn (editor), "RHIC design manual", revision of October 2000, http://www.rhichome.bnl.gov/NTshare/rhicdm/00_tocli.htm.

6. W. Fischer and $\bar{M}$. Blaskiewicz, "Luminosity increase at the incoherent beam-beam limit in RHIC", BNL C-A/AP/94 (2003).

7. V. Ptitsyn, private communication (2002).

8. D. Barton, PHOBOS; W. Cristie, STAR; M. Purschke, PHENIX; D. Beavis, BRAHMS; private communication (2002)

9. W. Herr and R. Paparella, "Landau damping of coherent beam-beam modes by overlap with synchrotron side-
Superbunch length $[\mathrm{m}]$

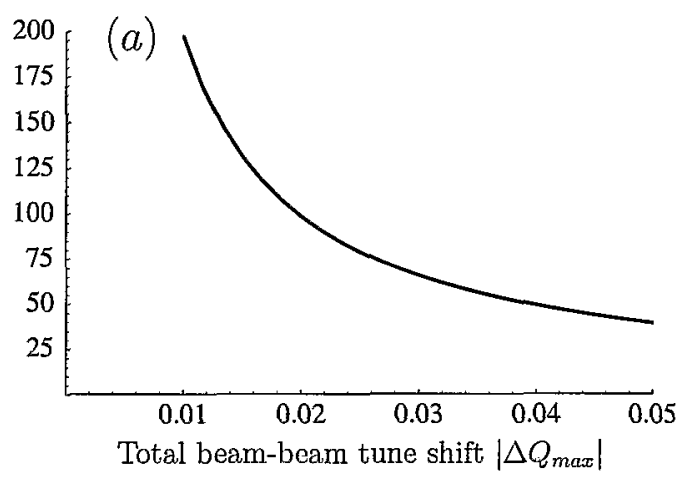

Luminosity $\left[\mathrm{cm}^{-2} \mathrm{~s}^{-1}\right]$

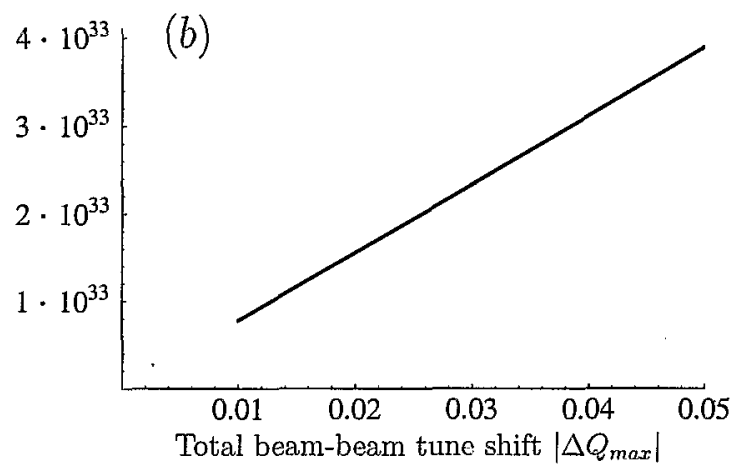

FIGURE 4. Superbunch length and luminosity per interaction point as a function of the total beam-beam tune shift $\left|\Delta Q_{\max }\right|$ in parts (a) and (b) respectively. Other parameters are given in Tab. 1.

bands", CERN LHC Project Note 304 (2002).

10. S. Peggs, "Beam-beam collisions and crossing angles in RHIC", proceedings of the LHC Beam-Beam Workshop at CERN 1999, BNL RHIC/AP/169 (1999).

11. M. Blaskiewicz and J.M. Brennan, "A barrier bucket experiment for accumulating de-bunched beam in the AGS", proceedings of the 1996 European Particle Accelerator Conference, Sitges, Spain (1996).

12. K.Y. Ng, "Multiple Injections with Barrier Buckets", proceedings of the 1998 European Particle Accelerator Conference, Stockholm, Sweden (1998).

13. M. Blaskiewicz, J.M. Brennan, T. Roser, K. Smith, R. Spitz, A. Zaltsman, M. Fujieda, Y. Iwashita, A. Noda, M. Yoshii, Y. Mori, C. Ohmori, Y. Sato, "Barrier Cavities in the Brookhaven AGS", proceedings of the 1999 Particle Accelerator Conference, New York (1999).

14. T. Bohl, T. Linnecar and E. Shaposhnikova, "Barrier buckets in the CERN SPS", proceedings of the 2000 European Particle Accelerator Conference, Vienna, Austria (2000).

15. M. Harrison, "High luminosity p-p operation at RHIC", BNL RHIC/AP/8 (1993). 